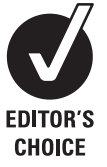

${ }^{1}$ Paediatric Haematology

Oncology Division, Department of Paediatrics, VU University

Medical Centre, Vrije University, Amsterdam, the Netherlands;

${ }^{2}$ Paediatric Haematology Oncology Division, Department of Paediatrics, Dr Sardjito Hospital, Gadjah Mada University, Yogyakarta, Indonesia; ${ }^{3}$ Department of Psycho-Social Research and Epidemiology, Dutch Cancer Institute-Antoni van Leeuwenhoek Hospital, Amsterdam, the Netherlands

Correspondence to:

S Mostert, Paediatric

Haematology Oncology Division, Department of Paediatrics, VU

University Medical Centre, Vrije University, Amsterdam,

the Netherlands:

s.mostert@vumc.nl

Accepted 14 July 2009

Published Online First

12 August 2009

\title{
Comparing childhood leukaemia treatment before and after the introduction of a parental education programme in Indonesia
}

\author{
S Mostert, ${ }^{1}$ M N Sitaresmi, ${ }^{2}$ C M Gundy, ${ }^{3}$ V Janes, ${ }^{1}$ Sutaryo, ${ }^{2}$ A J P Veerman ${ }^{1}$
}

\begin{abstract}
Setting: Previously, treatment and the results of treatment for childhood acute lymphoblastic leukaemia (ALL) in Indonesia differed significantly between poor and prosperous patients. Poor patients received less individual attention from oncologists and access to parental education and donated chemotherapy was lacking. Intervention: A structured parental education programme for both poor and prosperous parents was introduced in January 2004 to improve access to parental education and donated chemotherapy. The programme consisted of a video presentation, an information booklet, DVD, audiocassette, a statement-of-understanding for donated chemotherapy, and a complaints procedure. Informed consent was also sought.
\end{abstract}

Objective: Our study compared childhood ALL treatment outcome before and after the introduction of the parental education programme.

Design: The medical records of 283 children with $A L L$ diagnosed before (1997-2002; $n=164)$ and after (20042006; $n=119$ ) the introduction of the education programme were reviewed. Data on treatment results and parental socioeconomic status were collected.

Results: After the introduction of the education programme, treatment refusal decreased (from 14\% to $2 \%$ ) and event-free survival increased (from 13\% to 29\%) significantly among poor patients. Treatment dropout increased (from $0 \%$ to $13 \%$ ) significantly among prosperous patients. Overall, toxic death (from 23\% to $36 \%$ ) increased significantly, but there was no significant difference in event-free survival.

Conclusions: After introduction of the programme, treatment refusal decreased and event-free survival increased significantly among poor families. However, improved knowledge, skills and communication are still required to combat the high rates of toxic death and treatment dropout. Treatment intensity should be accompanied by improved supportive care.

The cure rate for childhood acute lymphoblastic leukaemia (ALL), the most common cancer in children, is about $80 \%$ in developed countries but is frequently less than $35 \%$ in developing nations. This difference is believed to be primarily due to treatment refusal or abandonment, a problem almost unknown in developed countries. ${ }^{12} \mathrm{~A}$ few countries have reported a reduction in treatment refusal or abandonment following the introduction of twinning programmes. In twinning, public hospitals in developing countries and established cancer centres elsewhere cooperate to improve survival among children with cancer. ${ }^{1-4}$ A twinning programme between Brazil and the USA

\section{What is already known on this topic}

- The cure rate for childhood acute lymphoblastic leukaemia is approximately $80 \%$ in developed countries, but is frequently less than $35 \%$ in developing countries.

- This difference is primarily due to treatment refusal or abandonment by poor patients in developing countries.

\section{What this study adds}

- Providing structured information about leukaemia, its treatment and donated chemotherapy to poor families can help to decrease treatment refusal and increase survival.

showed that installing a dedicated paediatric oncology unit, therapeutic protocols, trained nurses, $24 \mathrm{~h}$ on-site physician coverage and intensive care reduced the drop-out rate from $16 \%$ to $0.5 \%$ in 10 years. ${ }^{12} \mathrm{~A}$ twinning programme between Nicaragua and Italy showed that trained doctors and nurses, therapeutic protocols, research involvement and financial help for the construction of clinics and family homes reduced the dropout rate from $20 \%$ to $7 \%$ in 16 years. $^{23}$

In our twinning programme between Indonesia and the Netherlands, we found ${ }^{5}$ that treatment results in Indonesia differed significantly between 164 children with different socioeconomic backgrounds, even though the children were treated in the same hospital with the same protocol. Treatment refusal or abandonment occurred in $47 \%$ of 120 poor patients but in only $2 \%$ of 44 prosperous patients. Similarly, $11 \%$ of poor patients had an event-free survival compared with $45 \%$ of prosperous patients. There was a striking disparity in the way treatment was given to poor, as opposed to prosperous, patients. Poor patients received less individual attention from oncologists and parental education about leukaemia, its treatment and the possibility of receiving donated chemotherapy from a Dutch charity was lacking. ${ }^{5}$ There were no clear criteria for families to receive donated chemotherapy. It was assumed that oncologists informed all families that their children could receive part or all of their chemotherapy free, 
if required. However in practice, only 27 patients (16\%) were informed about this possibility. Most patients ( $\mathrm{n}=137,84 \%$ ) were not informed and had to pay for chemotherapy. Many poor parents could not afford the expensive medicines and treatment costs, and refused or abandoned treatment for their children. ${ }^{6}$

Starting in January 2004, a parental education programme for all parents, poor and prosperous, was introduced in Yogyakarta. This programme aimed to increase the access of poor patients to information about leukaemia, its treatment and donated chemotherapy. We hypothesised that increased access would decrease treatment refusal or abandonment and increase eventfree survival in poor patients. Because prosperous patients already had access to information about ALL from their personal oncologists and could afford the required chemotherapy before the introduction of the education programme, we did not expect their treatment results to differ much after introduction of the programme.

The objective of this study was to compare childhood leukaemia outcome before and after the introduction of the parental education programme.

\section{METHODS}

\section{Setting}

Indonesia has about 218 million inhabitants. As in other lowincome countries, there is a very large gap between the poor majority and the privileged minority. Literacy is around $92 \%{ }^{7}$ Indonesia has approximately 2000-3200 new childhood ALL cases annually. ${ }^{8}$ Our study was conducted in the academic Dr Sardjito Hospital in Yogyakarta, where 30-50 children are diagnosed with ALL each year. ${ }^{9}$ The paediatric department has four different types of wards: third, second, first and VIP. With increasing rank, ward conditions improve and the number of children per hospital room decreases. There are two types of polyclinic: general and VIP. Most patients attend second/third class wards (operated by junior residents who are supervised daily by oncologists) and the general polyclinic (staffed by junior residents with no experience in the field and only supervised at their own request). Patients who attend VIP/first class wards (operated by senior residents who are supervised daily by oncologists) and the VIP polyclinic (staffed by oncologists) have the same oncologist throughout treatment. In 2005 a limited health insurance card partly covering hospital costs for the poorest patients was introduced in Indonesia. However, government health insurance plans changed continuously and it was unclear which costs were covered. After the institution of health insurance, oncologists intensified treatment in 2005 and introduced a new higher intensity protocol in 2006. Oncologists hoped that adding more and higher doses of chemotherapy, now paid for by insurance, would decrease the relapse rate.

\section{Parental education programme}

Starting in January 2004, one social paediatrician provided the parental education programme at diagnosis. This paediatrician invited both parents to watch a video presentation of a paediatric oncologist explaining the disease and its treatment. Parents were encouraged to ask questions and the video presentation ensured that poor and prosperous parents received the same information. Parents were informed about chemotherapy donated by the Estella Foundation and clear rules were explained: all childhood ALL patients were entitled to receive methotrexate, vincristine, daunorubicin and L-asparaginase for free. US $\$ 0.04$ was charged for 6-mercaptopurine. Parents had to pay for dexamethasone (US\$0.04) themselves. In practice this meant that parents paid US $\$ 0.04$ instead of US\$1 per day for chemotherapy. (Parents still paid hospitalisation and polyclinic costs, paid for transportation, bone-marrow/lumbar punctures, radiographs, blood transfusions and antibiotics, and carried the cost of the loss of daily wages.) A complaints procedure was installed. Parents were given opportunities to communicate with or complain about donations to the Estella Foundation by mail or e-mail. Informed consent and Estella Foundation statements of understanding were introduced. Parents, patients, survivors and representatives of parent organisations encouraged parents to comply with treatment and not drop out. In addition to the video presentation, parents received an information booklet, DVD and audiocassette containing the same information. ${ }^{10}$

\section{Study design}

All children ( $0-16$ years) with newly diagnosed ALL were included. The medical records of patients diagnosed before (January 1997-August 2002) and after (January 2004-December 2006) the introduction of the parental education programme were studied.

Children were treated with the COM-ALL protocol (19971999), the WK-ALL protocol (1999-2005) or the Indonesia-ALL protocol (2005-2006). All protocols used the same risk stratification and consisted of induction ( 6 weeks), consolidation ( 5 weeks) and continuing therapy ( 96 weeks), with additional re-induction therapy ( 6 weeks) for high-risk patients. Total treatment takes 2 years.

Patient characteristics and treatment results were noted. Treatment failure was defined as refusal or abandonment of treatment, treatment-related death, or progressive or relapsed leukaemia. Treatment refusal was defined as rejection of any therapeutic intervention. Treatment abandonment was defined as therapy initiated but not completed. Our socioeconomic status classification identified children as coming from poor or prosperous families, based on two determinants: the monthly income level of the parents and the assigned hospital class during the diagnostic process. Both determinants are obtained routinely during hospital admission and recorded in medical records. The threshold of monthly income for poor versus prosperous families was set at US\$80 before and US\$100 after the introduction of the education programme. Patients attending VIP/first class wards and the VIP polyclinic were classified as prosperous. Patients attending second/third class wards and the general polyclinic were classified as poor.

The study was approved by the Medical Ethics Committee of Gadjah Mada University.

\section{Data analysis}

Data management and analysis were performed using SPSS v 12. Differences in patient characteristics and treatment outcome before and after the introduction of the education programme were compared using the $\chi^{2}$ test, Fisher's exact test, the t test and the $z$ test. Only outcome during first 2 years (duration to complete treatment) was considered in this comparison to avoid bias caused by different follow-up times before and after the introduction of the parental education programme. The probability of event-free survival was estimated using the Kaplan-Meier method; estimates were compared using the logrank test. Event-free survival was measured from the date when the patient received the diagnosis of leukaemia to first 
Table 1 Outcome at 2 years after diagnosis for the overall population before $(n=164)$ and after $(n=119)$ introduction of the parental education programme

\begin{tabular}{llll}
\hline & $\begin{array}{l}\text { Before introduction of } \\
\text { the parental education } \\
\text { progmme }(\mathbf{n}=164)\end{array}$ & $\begin{array}{l}\text { After introduction of the } \\
\text { parental education } \\
\text { progmme (n=119) }\end{array}$ & $\begin{array}{l}\text { p Value comparing the } \\
\text { two periods }\end{array}$ \\
\hline Treatment refusal & $18(11 \%)$ & $3(3 \%)$ & 0.010 \\
Treatment abandonment & $39(24 \%)$ & $28(24 \%)$ & $\mathrm{NS}$ \\
Treatment-related death & $38(23 \%)$ & $43(36 \%)$ & 0.017 \\
Progressive or relapsed leukaemia & $30(18 \%)$ & $8(7 \%)$ & 0.005 \\
Event-free survival & $39(24 \%)$ & $37(31 \%)$ & $\mathrm{NS}$ \\
\hline
\end{tabular}

treatment failure (refusal or abandonment of treatment, treatment-related death, progressive or relapsed leukaemia) or the date of the last follow-up. The most recent follow-up on treatment outcome was conducted in December 2008. Cox proportional hazards model evaluated the effect of treatment period, socioeconomic status, risk stratification and gender on risk of treatment failure.

\section{RESULTS}

We reviewed the medical records of 283 children with ALL diagnosed before and after the introduction of the parental education programme.

From January 1997 to August 2002 (before the introduction of the education programme), 164 patients received a diagnosis of ALL. Twenty one (13\%) patients did not start therapy, 36 (22\%) followed the COM-ALL protocol and 107 (65\%) followed the WK-ALL protocol.

From January 2004 to December 2006 (after the introduction of the education programme), 119 patients received a diagnosis of ALL. Six patients (5\%) did not start therapy, $74(62 \%)$ followed the WK-ALL protocol, and 39 (33\%) followed the Indonesia-ALL protocol.

No significant differences in age, gender, socioeconomic status or risk stratification were found in children before $(n=164)$ and after $(n=119)$ the introduction of the education programme.

Table 1 shows outcome at 2 years after diagnosis in the overall population before $(n=164)$ and after $(n=119)$ the introduction of the parental education programme. Treatment refusal in the overall population decreased significantly $(p=0.010)$ after the introduction of the programme. Treatment abandonment was not significantly different between the two periods. Treatmentrelated death increased significantly $(p=0.017)$ after the introduction of the programme. No significant difference in event-free survival estimates in the overall population was found between the periods before and after the introduction of the programme. Hazard ratios for treatment failure were 1.4 (95\% CI 1.1 to 1.8) for the early versus recent period, 2.6 (95\% CI 1.8 to 3.7$)$ for the poor versus prosperous population, 1.8 (95\% CI 1.4 to 2.4) for high-risk versus standard-risk ALL, and 0.9 (95\% CI 0.7 to 1.2$)$ for male versus female gender.

Table 2 shows outcome at 2 years after diagnosis in the poor population before $(n=120)$ and after $(n=96)$ the introduction of the parental education programme. Treatment refusal in the poor population decreased significantly $(p=0.001)$ after the introduction of the programme: $2 \%$ of 96 poor patients refused treatment as opposed to $14 \%$ of 120 poor patients in the previous period. The difference in treatment abandonment between the two periods was not significant. Figure 1 shows the significant improvement in the event-free survival estimate in the poor population after the introduction of the education programme $(p=0.004)$.

Table 3 shows outcome at 2 years after diagnosis in the prosperous population before $(n=44)$ and after $(n=23)$ the introduction of the parental education programme. The difference in treatment refusal between the two periods was not significant. Treatment abandonment in the prosperous population increased significantly $(p=0.037)$ after the introduction of the programme: $13 \%$ of 23 prosperous patients abandoned treatment as opposed to none of the 44 prosperous patients in the previous period. Figure 2 shows no significant difference in event-free survival estimates in the prosperous population before and after introduction of the education programme.

\section{DISCUSSION}

Before the introduction of the structured parental education programme, information about leukaemia, its treatment and the availability of external funding was seldom given to poor families. Resident doctors provided information to the poor, but they lacked experience in paediatric oncology and knowledge about funds, and consequently, information was incomplete. In addition, strong social hierarchical structures hindered communication between doctors and poor parents. As a result, many parents did not understand why it was important for children to take their medication daily for 2 years. ${ }^{5}$ Most parents could not afford the prolonged treatment costs and had no access to donated chemotherapy. ${ }^{6}$ Not all health care providers believed it was possible to cure poor patients with leukaemia, as expensive and prolonged treatment is required. ${ }^{5}$ Most health care

Table 2 Outcome at 2 years after diagnosis in the poor population before $(n=120)$ and after $(n=96)$ introduction of the parental education programme

\begin{tabular}{llcl}
\hline & $\begin{array}{l}\text { Before introduction of } \\
\text { the parental education } \\
\text { programme } \mathbf{( n = 1 2 0 )}\end{array}$ & $\begin{array}{l}\text { After introduction of the } \\
\text { parental education } \\
\text { programme }(\mathbf{n}=\mathbf{9 6})\end{array}$ & $\begin{array}{l}\mathbf{p} \text { Value comparing the } \\
\text { two periods }\end{array}$ \\
\hline Treatment refusal & $17(14 \%)$ & $2(2 \%)$ & 0.001 \\
Treatment abandonment & $39(33 \%)$ & $25(26 \%)$ & NS \\
Treatment-related death & $30(25 \%)$ & $34(35 \%)$ & NS \\
Progressive or relapsed leukaemia & $18(15 \%)$ & $7(7 \%)$ & NS \\
Event-free survival & $16(13 \%)$ & $28(29 \%)$ & 0.004 \\
\hline
\end{tabular}


Figure 1 Kaplan-Meier estimates of event-free survival in the poor population before $(n=120)$ and after $(n=96)$ introduction of the parental education programme $(p=0.004)$. Events included refusal or abandonment of treatment, treatment-related death and progressive or relapsed leukaemia.

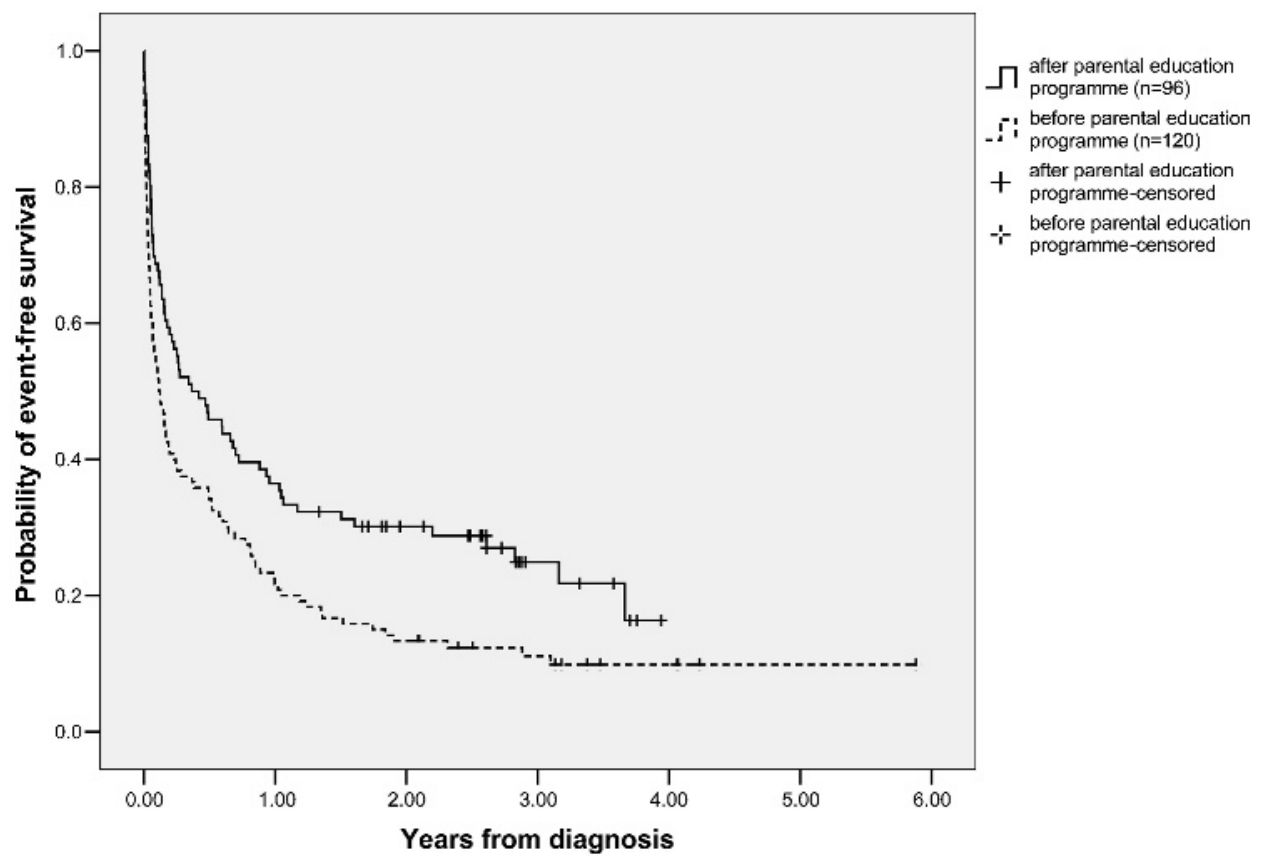

providers believed that prosperous patients complied better with treatment and that doctors provided superior treatment for richer patients. We warned of a self-fulfilling prophecy: if doctors expect poor families to perform less well, their own input may decrease and subsequently parental and patient compliance may diminish leading to worse performance and treatment results. ${ }^{11}$

Starting in January 2004, a parental education programme for all parents, poor and prosperous, was introduced to increase the access of poor patients to information about leukaemia, its treatment and donated chemotherapy. ${ }^{6}$ We hypothesised that increased access would significantly decrease treatment refusal and abandonment and increase survival in poor patients. Our study partially confirmed this hypothesis. After the introduction of the education programme, treatment refusal decreased (from $14 \%$ to $2 \%$ ) significantly, treatment abandonment decreased (from $33 \%$ to $26 \%$ ) but not significantly, and event-free survival improved (from $13 \%$ to $29 \%$ ) significantly among poor children. Therefore, it appears our parental education programme informed and encouraged parents to start treatment; showing interest, investing time, being willing to give information, and providing donated chemotherapy to poor families increase compliance and improve the survival of poor patients.

Because prosperous patients already had access to information about ALL from their personal oncologists and could afford the required chemotherapy before the introduction of the education programme, we did not expect their treatment outcome to differ much after introduction of the programme. However, we did find that prosperous patients abandoned treatment more frequently (from $0 \%$ to $13 \%$ ) after the introduction of the programme. This may be due to the fact that after the institution of limited health insurance in 2005, oncologists intensified treatment, thereby increasing the toxicity of treatment. From interviews with families who dropped out, ${ }^{12}$ we learned that prosperous patients abandoned treatment due to a fear of toxicity; after seeing severe side-effects in their child or observing other children die on wards after having received chemotherapy, parents decided to stop treatment.

Treatment-related death increased (from 23\% to $36 \%$ ) significantly in the overall population. Several factors may have contributed to this: (1) with fewer patients dropping out, more patients were at risk of dying of treatment-related toxicity; (2) the use of intensified and more toxic chemotherapy leads to an increased chance of toxic death if appropriate supportive care facilities are not available; the poor nutritional status of most patients results in decreased tolerance to chemotherapy, alters the metabolism of chemotherapy, and is associated with an increased infection rate, resulting in poor clinical outcome. ${ }^{13}$ Doctors must improve their knowledge and skills to detect and vigorously treat complications as early as possible and treatment intensity should be accompanied by improved supportive care ${ }^{10}{ }^{14}$; and (3) there has been a recent increase in the numbers of patients admitted to hospital, resulting in immune-compromised leukaemia patients in overcrowded hospital rooms being

Table 3 Outcome at 2 years after diagnosis in the prosperous population before $(n=44)$ and after $(n=23)$ introduction of the parental education programme

\begin{tabular}{|c|c|c|c|}
\hline & $\begin{array}{l}\text { Before introduction of the parental } \\
\text { education programme }(n=44)\end{array}$ & $\begin{array}{l}\text { After introduction of the parental } \\
\text { education programme }(n=23)\end{array}$ & $\begin{array}{l}\mathrm{p} \text { Value comparing the two } \\
\text { periods }\end{array}$ \\
\hline Treatment refusal & $1(2 \%)$ & $1(4 \%)$ & NS \\
\hline Treatment abandonment & $0(0 \%)$ & $3(13 \%)$ & 0.037 \\
\hline Treatment-related death & $8(18 \%)$ & $9(39 \%)$ & NS \\
\hline Progressive or relapsed leukaemia & $12(27 \%)$ & $1(4 \%)$ & 0.026 \\
\hline Event-free survival & $23(52 \%)$ & $9(39 \%)$ & NS \\
\hline
\end{tabular}


Figure 2 Kaplan-Meier estimates of event-free survival among the prosperous population before $(n=44)$ and after $(n=23)$ introduction of the parental education programme $(p=N S)$. Events included refusal or abandonment of treatment, treatment-related death, and progressive or relapsed leukaemia.

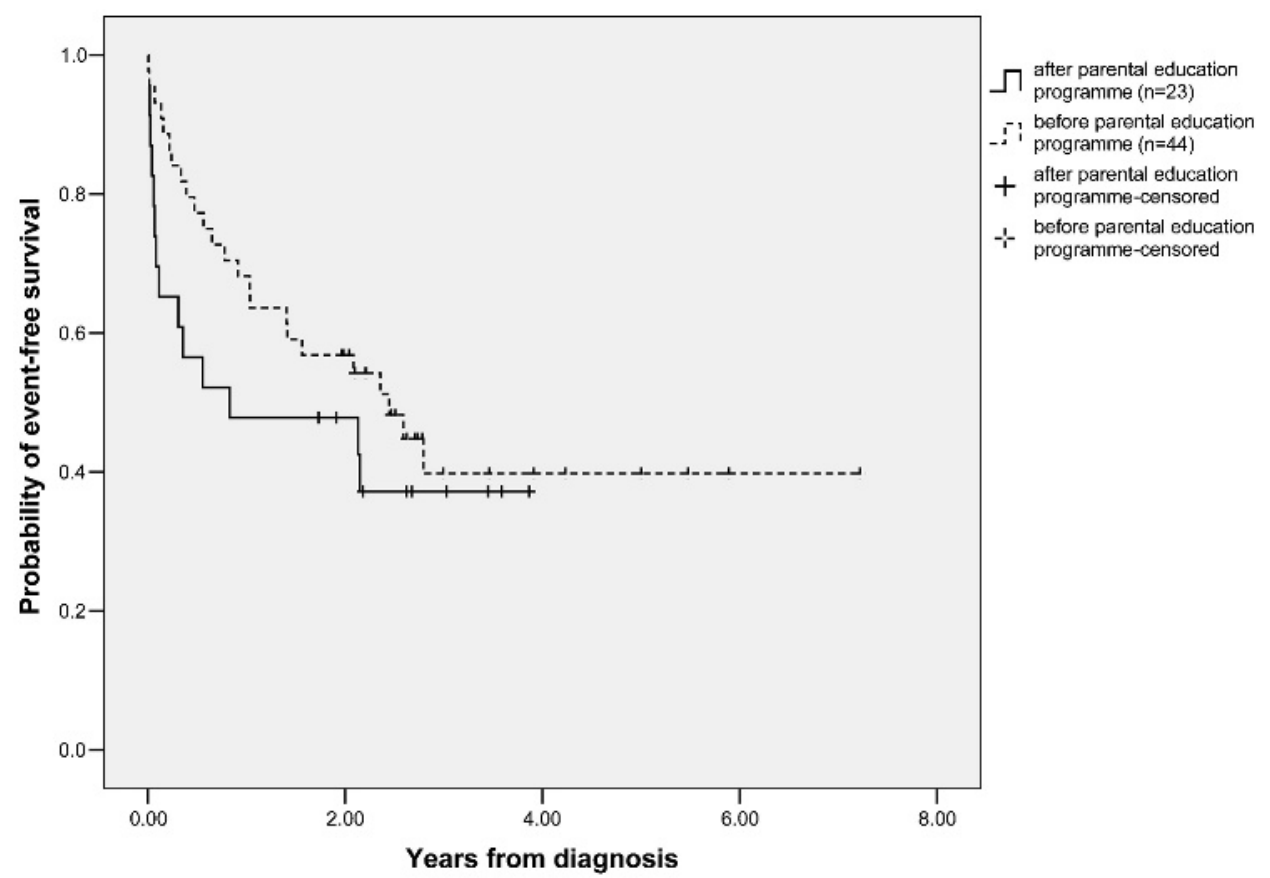

more at risk of developing potentially fatal infections. To solve this problem, a new paediatric oncology ward will be built with the help of charities.

Although treatment refusal in the poor and overall population decreased significantly, substantial numbers of patients $(27 \%)$ still drop out. Our previous study ${ }^{12}$ where families who dropped out were interviewed, showed that the reasons for dropping out were complex and included financial problems, transportation difficulties, beliefs about the incurability of ALL, fear and the experience of severe side-effects, and dissatisfaction with doctors. Parents who refused treatment had health beliefs based on "lay resources" such as the stories of relatives, friends or other parents. Unfortunately, they went home immediately after diagnosis before being exposed to the education programme. In contrast, parents who abandoned treatment initially trusted information presented in the education programme. However, after their child had experienced severe side-effects and they had seen other ALL patients die after receiving chemotherapy, they started to evaluate the benefits of chemotherapy. From the families who refused treatment, we learned that education programmes should be initiated during diagnostic evaluation. From the families who abandoned treatment, we learned that education programmes should be an ongoing process, with regular group meetings of parents and professionals, to discuss health beliefs, fears and experiences on wards. ${ }^{12}$ Communication with all families, poor and prosperous, is essential. Doctors themselves have an important influence on the compliance, drop-out and survival of children with ALL. ${ }^{1}{ }^{15-18}$

To improve survival, toxic death and patient drop-out must decrease as causes of treatment failure. Health care providers themselves need an education programme to improve: (1) their knowledge and skills in handling treatment toxicity; and (2) their attitudes and communication skills in order to prevent treatment abandonment.

Historical analyses have weaknesses. Without controls or randomisation, they cannot really demonstrate that results are only due to the intervention in question, in our case the parental education programme. However, a few considerations are worthy of mention: the introduction of a limited health insurance card for the poorest patients in 2005 may have encouraged some poor families to follow treatment; the intensified treatment in 2005 and 2006 may have contributed to treatment-related death and treatment abandonment; more families may have been able to access treatment with the end of the 1997 Asian economic crisis; the recent long hospital waiting lists may have influenced treatment results; and doctors may have improved or disimproved over time. Also, the small number of prosperous patients limits the interpretation of our results.

We conclude that after the introduction of the parental education programme, poor families received structured information about leukaemia, its treatment and donated chemotherapy for the first time. Treatment refusal decreased and survival increased significantly among poor patients. However, work remains to be done. To improve survival, it is important that toxic death and treatment abandonment decrease as causes of treatment failure. Improvement in the knowledge, skills and communication of doctors is required to manage treatment toxicity and prevent treatment abandonment. Treatment intensity should be matched with appropriate supportive care.

Acknowledgements: We thank Drs Jaap de Vries for linguistic assistance and Ignatius Purwanto for secretarial assistance.

Funding: Our project is supported by grants from the Dutch Cancer Society Koningin Wilhelmina Fonds, and the Estella Foundation.

Competing interests: None.

Ethics approval: This study was approved by the Medical Ethics Committee of Gadjah Mada University.

Provenance and peer review: Not commissioned; externally peer reviewed.

\section{REFERENCES}

1. Howard SC, Pedrosa M, Lins M, et al. Establishment of a pediatric oncology programme and outcomes of childhood acute lymphoblastic leukemia in a resourcepoor area. JAMA 2004;291:2471-5.

2. Arora RS, Eden T, Pizer B. The problem of treatment abandonment in children from developing countries with cancer. Pediatr Blood Cancer 2007:49:941-6. 
3. Masera G, Baez F, Biondi A, et al. North-South twinning in paediatric haematooncology: the La Mascota programme, Nicaragua. Lancet 1998;352:1923-6.

4. Harif M, Barsaoui S, Benchekroun S, et al. Treatment of childhood cancer in Africa. Preliminary results of the French-African paediatric oncology group. Arch Pediatr 2005; 12:851-3.

5. Mostert S, Sitaresmi MN, Gundy CM, et al. Influence of socioeconomic status on childhood acute lymphoblastic leukemia treatment in Indonesia. Pediatrics 2006;118:e1600-6.

6. Mostert S, Sitaresmi MN, Gundy CM, et al. Does aid reach the poor? Experiences of a childhood leukaemia outreach-programme. Eur J Cancer 2008;45:414-19.

7. Departemen Kesehatan Republik Indonesia. Ministry of Health Republic of Indonesia. See http://www.depkes.go.id (accessed 11 September 2009).

8. Sutaryo, Sumadiono, Suhadi, et al. The Pilot Protocol of Wijaya Kusuma Acute Lymphoblastic Leukemia of Childhood 1999. Indonesian Multicentre Study. Yogyakarta: Gadjah Mada University Press, 1999.

9. Sutaryo, Sumadiono, Suhadi, et al. The Protocol of Wijaya Kusuma Acute Lymphoblastic Leukemia of Childhood 2000. Indonesian Multicentre Study. Yogyakarta: Gadjah Mada University Press, 2000.

10. Lilleyman J. Simple deliverable therapy needed for childhood leukaemia. Lancet 2003;362:676-7.
11. Mostert S, Sitaresmi MN, Gundy CM, et al. Attitude of health-care providers toward childhood leukemia patients with different socio-economic status. Pediatr Blood Cancer 2008;50:1001-5.

12. Sitaresmi MN, Mostert S, Schook R, et al. Treatment refusal and abandonment in childhood acute lymphoblastic leukemia in Indonesia: an analysis of causes and consequences. Psychooncology 2009 May 11 [Epub ahead of print].

13. Sala A, Pencharz P, Barr RD. Children, cancer, and nutrition - a dynamic triangle in review. Cancer 2004;100:677-87.

14. Hesseling PB, Broadhead R, Molyneux E, et al. Malawi pilot study of Burkitt lymphoma treatment. Med Pediatr Oncol 2003;41:532-40.

15. Lilleyman JS, Lennard L. Non-compliance with oral chemotherapy in childhood leukaemia. An overlooked and costly cause of late relapse. BMJ 1996;313:1219-20.

16. Tebbi CK. Treatment compliance in childhood and adolescence. Cancer Supp/ 1993:71:3441-9

17. Mostert S, Sitaresmi MN, Gundy CM, et al. Parental experiences of childhood leukemia treatment in Indonesia. J Pediatr Hematol Oncol 2008;30:738-43.

18. Israels T, Chirambo $\mathrm{C}$, Caron $\mathrm{H}$, et al. The guardians' perspective on pediatric cancer treatment in Malawi and factors affecting adherence. Pediatr Blood Cancer 2008:51:639-42.

\section{Archivist}

\section{Maternal genitourinary infection and cerebral palsy}

A meta-analysis of 19 studies in 2000 showed that symptomatic chorioamnionitis in pregnancy was associated with a fivefold increase in risk of cerebral palsy among term infants and a twofold increase among preterm infants. The effect of urinary tract infections in pregnancy on this risk is uncertain. Now a US study (Joshua R Mann and colleagues. Dev Med Child Neurol 2009;51:282-8) has suggested that the increased risk of cerebral palsy associated with maternal genitourinary infection may be confined to preterm, low birthweight infants and infection in the first or second trimesters.

The study used data from Medicaid billing records for South Carolina in 1996-2002. Maternal genitourinary infections included International Classification of Diseases version 9 (ICD-9) codes for trichomoniasis, gonorrhoea, Chlamydia trachomatis/non-gonococcal urethritis, vulvovaginal candidiasis, urinary tract infection, vaginitis, cervicitis, upper reproductive tract infections, and unspecified "infections of the genitourinary tract during pregnancy". Overall, maternal genitourinary infection during pregnancy was associated with a significant $27 \%$ increase in risk of cerebral palsy. On further analysis the increase in risk was shown to be confined to infections in the first two trimesters and infants born before 27 weeks and with a birth weight of $<2500$ g. The clearest association was with chlamydial infection, which doubled the risk. Urinary tract infection was associated with a significant $25 \%$ increase in risk and trichomoniasis with a highly significant $74 \%$ increase. With a more restricted definition of confirmed cerebral palsy only chlamydial infection was associated with increased risk. There was no association between gonorrhoea or candidiasis and cerebral palsy.

Chlamydial infection, and possibly trichomoniasis or urinary tract infection in the first two trimesters of pregnancy, increases the risk of cerebral palsy among preterm or low birthweight infants. 